\title{
SPECIAL ISSUE \\ EVENT-INDUCED THETA RESPONSES AS A WINDOW ON THE DYNAMICS OF MEMORY
}

\author{
Marcel Bastiaansen and Peter Hagoort \\ (Max Planck Institute for Psycholinguistics, and F.C. Donders Centre for Cognitive \\ Neuroimaging Nijmegen, The Netherlands)
}

\begin{abstract}
An important, but often ignored distinction in the analysis of EEG signals is that between evoked activity and induced activity. Whereas evoked activity reflects the summation of transient post-synaptic potentials triggered by an event, induced activity, which is mainly oscillatory in nature, is thought to reflect changes in parameters controlling dynamic interactions within and between brain structures. We hypothesize that induced activity may yield information about the dynamics of cell assembly formation, activation and subsequent uncoupling, which may play a prominent role in different types of memory operations. We then describe a number of analysis tools that can be used to study the reactivity of induced rhythmic activity, both in terms of amplitude changes and of phase variability.

We briefly discuss how alpha, gamma and theta rhythms are thought to be generated, paying special attention to the hypothesis that the theta rhythm reflects dynamic interactions between the hippocampal system and the neocortex. This hypothesis would imply that studying the reactivity of scalp-recorded theta may provide a window on the contribution of the hippocampus to memory functions.

We review studies investigating the reactivity of scalp-recorded theta in paradigms engaging episodic memory, spatial memory and working memory. In addition, we review studies that relate theta reactivity to processes at the interface of memory and language. Despite many unknowns, the experimental evidence largely supports the hypothesis that theta activity plays a functional role in cell assembly formation, a process which may constitute the neural basis of memory formation and retrieval. The available data provide only highly indirect support for the hypothesis that scalp-recorded theta yields information about hippocampal functioning. It is concluded that studying induced rhythmic activity holds promise as an additional important way to study brain function.
\end{abstract}

Key words: EEG, oscillations, theta rhythm, memory, hippocampus

\section{INTRODUCTION}

Cognitive neuroscience has profited tremendously from hemodynamic imaging techniques such as PET and fMRI in linking human cognition to brain structures. Until PET and fMRI became available, we had to rely largely on the experiments of nature in the form of neuropsychological deficits. These new imaging techniques made it possible to investigate in the living, intact human brain the neural correlates of complex cognitive functions such as memory, language, attention and perception under experimentally well-controlled conditions. This has resulted in a wealth of data on the neural architectures of a variety of cognitive functions.

One thing that has become very clear on the basis of these imaging data is that a one-to-one mapping between a brain area and a specific component of a 
cognitive function is very often far too simplistic. Activations of specific areas are often reported in imaging studies on different tasks or cognitive functions. It seems that individual cortical areas can be recruited dynamically in more than one functional network. Mesulam (1998) refers to this aspect of neural organization as the principle of selectively distributed processing: many cortical areas operate as a node in multiple functional networks. This raises the issue of how, for a given function, the dynamic recruitment of the participating cortical and subcortical areas takes place.

The dynamics of neural activity is often better captured by imaging methods with a high temporal resolution such as EEG and MEG than by imaging methods that are limited by the relatively slow time constant of the hemodynamic response. The measurement of Event-Related brain Potentials or Fields (ERPs, ERFs) has provided a wealth of fine-grained information about the time course of cognitive processing events (e.g. Van Turennout et al., 1998). However, ERPs and ERFs have not been very informative about the dynamics of functional network formation since they do not capture the transitions in network recruitment very well. In this context, recent years have seen an increasing interest in another aspect of EEG and MEG activity, on which we will focus in this paper. This aspect is related to patterns of synchronization and desynchronization that are observed in the electrophysiological activity of the brain. Interest in these phenomena has developed as a result of the view that they might provide a window on the dynamics of cell assembly formation, by which spatially distributed brain areas become linked together in dynamic networks involved in sensory integration, object representation and memory encoding or retrieval.

Synchronization and desynchronization of the brain's electrophysiological activity is observed in relation to different aspects of cognition. It is, therefore, often referred to as Event-Related Synchronization (ERS) and Event-Related Desynchronization (ERD; Pfurtscheller and Aranibar, 1977). We prefer to refer to both as event-related reactivity of frequency-specific EEG rhythms. This eventrelated reactivity can occur in different frequency bands. So far, most of the attention has been directed to the theta, alpha and gamma frequency bands. With respect to memory functions, the power increase in the theta band seems to be particularly relevant. This corresponds with the widely accepted assumption that the hippocampus is one of the key structures in the generation of the theta rhythm.

Although much is still unclear about the functional role of brain rhythmicity, sufficient knowledge has been acquired to justify the proposition that it plays a role in dynamic network formation. In this paper, we will illustrate how certain aspects of memory formation and retrieval are reflected in modulations of the theta rhythm. Although it is not our claim that these results provide a completely new perspective on memory processes, we believe that the types of analyses that we discuss in this paper might give new insights into the dynamics of memory formation and retrieval. In the remainder of this paper we will describe in some detail the nature of the brain activity on which the analyses are based, and the way in which the analyses are done. Furthermore, we discuss the brain structures known to be involved in the generation of the theta-rhythm, and finally we discuss relevant results in relation to memory. 


\section{EVOKED AND INDUCED EEG ACTIVITY}

In order to understand the rationale behind the analyses of event-related changes in induced band power (IBP), it is important to distinguish between two types of activities in EEG or MEG: induced activity and evoked activity. Evoked activity may be defined as activity that is directly driven by stimuli or state changes. Evoked activity is both time-and phase-locked to the eliciting event, because each time the event occurs similar EEG activity is produced (evoked) by the stimulus. Therefore evoked activity can be extracted from the ongoing EEG by a straightforward averaging of the event-related EEG epochs. The resulting average is a so-called Event-Related Potential (ERP). Note that this activity is not necessarily rhythmic in nature. A well-known example of evoked activity is the N1/P2 complex elicited by the presentation of auditory stimuli.

Induced activity, on the other hand, is largely rhythmic (oscillatory) in nature, and may be defined as oscillations caused or modulated by stimuli or state changes that do not directly drive the rhythm (after Bullock, 1992, pp.1). In other words, induced activity is best considered as rhythmic background activity that is modulated, rather than evoked, by external (i.e. some sort of stimulation) or internal (e.g., motor preparation, cognitive processing) events. The phase of an induced response can differ from trial to trial (see also Tallon-Baudry and Bertrand, 1999). As a result, induced activity is time-locked, but not necessarily phase-locked to the event. Since straightforward averaging leads to phase cancellation, induced activity will be 'averaged out' of the ERP as if it were noise. The important implication here is that the neurophysiological dynamics thought to underlie induced activity cannot be captured with standard ERP methodology. Different methods are needed in order to quantify the relevant parameters of changes in induced activity, as we will see in section 1.2. A wellknown example of an induced rhythmic response is the amplitude attenuation of the occipital alpha rhythm by the opening of the eyes (Berger, 1929).

\subsection{Neurophysiological Dynamics Underlying Induced Activities}

It has been proposed (Pfurtscheller and Lopes da Silva, 1999) that the two distinct types of activities are related to differences in the underlying neurophysiological dynamics (for supportive empirical evidence, see e.g. Klimesch et al., 2000; Bastiaansen and Brunia, 2001). Evoked activity as indexed by ERPs mainly reflects the summation of post-synaptic potentials impinging on local ensembles of cortical pyramidal neurons. Cortical pyramidal neurons are aligned in their orientation with respect to the cortical surface. Synchronous activation of these neurons, therefore, results in a field change that can be detected at a distance, i.e. the scalp. The neurons in subcortical areas such as the hippocampus are often not well aligned, and their activity thereby results in socalled closed fields that are undetectable at a distance. For this reason scalprecorded ERPs are cortical in nature, reflecting the contribution of cortical areas that are active at a specific time during the execution of a certain task.

Induced activity is similar to evoked activity in the sense that both are only 'visible' through the impact of neural activity on the cortical pyramidal neurons. 
However, the crucial difference between evoked and induced activity is that the latter especially reflects functional changes in the parameters controlling dynamic interactions within and between brain structures (Pfurtscheller and Lopes da Silva, 1999). These interactions can be subcortico-cortical or cortico-cortical in nature. Induced activity reflects dynamic processes involved in both the formation and the activation of spatially distributed, yet functionally coherent cell assemblies. As such, induced activity may be functionally related to processes such as long-range integration, feature binding or the formation of memories (see section 2). Additionally, in special cases such as the alpha rhythm induced activity can provide a window on the influences of specific subcortical structures on cortical activity (see section 2.1). In general, as we will emphasize throughout this paper, analyzing induced activity may allow one to empirically test hypotheses that are quite different from those obtained with ERPs.

Functional changes in induced activity, as expressed in the event-related reactivity of frequency-specific EEG rhythms such as theta, alpha or gamma, can be evaluated on two dimensions: their amplitude (cf. section 1.2.1) and their phase variability (cf. section 1.2.2). For the alpha frequency band, the eventrelated reactivity usually results in a reduction in the induced activity (see section 2.1), while for the other frequency bands the event-related reactivity is usually associated with an increase in induced activity. In general, it can be said that amplitude increases in induced activity reflect an increase in synchrony of the underlying neural populations.

Changes in phase variability, on the other hand, may reflect the processes of the formation and active uncoupling of cell assemblies. Here the hypothesis is that the formation of a cell assembly is realized through a transient phase-locking of EEG oscillations. We distinguish between two types of phase-locking. The first type of phase-locking, termed phase synchronization (e.g. Lachaux, Rodriguez, Martinerie et al., 1999), involves a transient event-related phase synchronization between two brain areas (but not necessarily phase-locked to the stimulus). A second type of phase-locking occurs when the presentation of a stimulus leads to a resetting of the phase of an EEG/MEG oscillation (e.g. Friston et al., 1997; Rizzuto et al., 2001). Note that when such a transient phase-resetting occurs, the distinction between evoked and induced activity, as defined in the previous section, becomes problematic. Strictly speaking, one could argue that since the phase resetting is evoked by the stimulus, the induced activity 'becomes' evoked activity for a short period of time. This indicates that the distinction between evoked activity and induced activity is a relative distinction rather than an absolute one. Note that some authors have argued that specific ERP components, such as the P300, may be a result of a transient event-related phase-resetting of induced rhythmic activity (Basar et al., 2001).

While phase synchronization and phase-resetting may signal the formation of a cell assembly, active uncoupling of a cell assembly, that is, the disruption of synchronized neuronal firing, may be reflected by a transient period of phasescattering (see Lachaux et al., 2000, for a similar proposition). Recently, convincing experimental evidence has been reported, supporting this notion (Rodriguez et al., 1999). 


\subsection{Tools for Analyzing Induced Activity}

The distinction between evoked and induced activity has implications for the analysis of EEG/MEG signals. As we noted, evoked activity may be extracted from the raw EEG by straigthforward averaging of single epochs. However, this approach does not apply to induced activity. Since straightforward averaging leads to phase cancellation, induced activity will be 'averaged out' of the ERP as if it were noise. In the following we give a short description of the available methodologies that can be used to extract induced activity from the raw EEG. Note that we do not intend to give an exhaustive review of all the technical issues and problems associated with the proposed analysis tools. Rather, our intention is merely to give the reader an outline of the possible avenues for the analysis of induced EEG/MEG activity.

\subsubsection{Quantifying Amplitude Changes of Induced Activity}

In order to quantify amplitude changes of induced activity, one needs a procedure that extracts the activity that is not phase-locked from the raw EEG. The most elementary way of accomplishing this involves the computation of task-related power (e.g. Gevins et al., 1997). Here the power spectrum of the EEG measured during one condition is compared to that in another condition (the resting, or control condition). This approach gives a good picture of the overall changes in signal power, but it does not yield any information about the evolution of power over time. Therefore, a measure of event-related power changes may be preferred over measures that have the poor temporal resolution of blocked designs. Two ways of quantifying the temporal evolution of power have been described in the literature: Induced Band Power (IBP) analysis and single-trial wavelet analysis.

IBP analysis was first proposed by Kalcher and Pfurtscheller (1995). The procedure for calculating IBP changes is the following: (i) single EEG epochs are bandpass filtered; (ii) these filtered epochs are averaged, yielding a bandpass filtered ERP that corresponds to the pure phase-locked activity in that frequency band; (iii) from each single, bandpass filtered EEG epoch, the bandpass filtered ERP is subtracted, thereby strongly attenuating the phaselocked activity from the non-phase-locked activity; (iv) the power of the subtracted single-trial data is computed, preferably by means of a signal envelope (Clochon et al., 1996); (v) averages over the single-trial power are then computed, and the changes in induced band power are expressed as a percentage of the power in a baseline interval (see e.g. Klimesch, 1999 for a detailed description of this approach).

One point that must be emphasized concerns the time resolution of IBP analyses. Although some authors have claimed that IBP yields a millisecond time resolution when it is computed using a signal envelope (Clochon et al., 1996), a recent simulation study has shown that this is not the case (Knosche and Bastiaansen, 2002). This study reveals that the time resolution of IBP roughly corresponds to half a period of the slowest frequency component contained in the signal. For example, for theta activity (usually defined as ranging from 4-7 Hz), 
this would be $125 \mathrm{~ms}$ (one period of a sine wave at $4 \mathrm{~Hz}$ lasts $250 \mathrm{~ms}$ ). It should be noted that this relatively poor temporal resolution may lead to the IBP changes that precede the actual signal change, if the latter has a sudden onset (see Knosche and Bastiaansen, 2002 for examples and details).

Wavelet analysis is to some extent equivalent to IBP analysis. Therefore, we discuss only IBP analysis here. For a clear and concise description of single-trial wavelet analysis, the reader is referred to Tallon-Baudry and Bertrand (1999) or Samar et al., (1999). However, there are a number of differences between IBP and wavelets that we briefly mention here. First, in the original outlines of IBP analysis (Kalcher and Pfurtscheller, 1995; Klimesch et al., 1998), the temporal resolution of the IBP is the same for all frequencies, while wavelet analysis provides a temporal resolution that improves with higher frequencies. This disadvantage of IBP can, however, easily be overcome by using a signal envelope rather than the squared signal amplitude as an estimate of the power in a particular frequency band (cf. Clochon et al., 1996; Knosche and Bastiaansen, 2002). Second, with wavelet analysis information about the phase of the signal is retained (although most reports of wavelet analysis use time-frequency plots in which the phase information is not used). In contrast, IBP measures do not contain phase information. Third, because of the subtraction procedure used in IBP computation, IBP tends to underestimate the magnitude of the oscillatory activity if it is phaselocked to the stimulus.

\subsubsection{Quantifying Phase Relations of Induced Activity}

The analysis of changes in the phase relationship of an induced rhythm can focus on two aspects. First, when an EEG signal enters into (more or less precise) phase-locking with a stimulus, or a cognitive process captured by an experimental condition, this phenomenon may be termed phase-resetting. The reverse process is termed phase-scattering. As we hypothesized above, these phase-sensitive processes may reflect the dynamic formation, and the subsequent uncoupling, of a cell assembly or functional network. The amount of phase consistency can be roughly visualized by applying an appropriate bandpass filter to the average ERP, leaving intact the frequency band of interest. The extent to which oscillatory components are present in the average ERP then indicates the amount of phase consistency over trials, since averaging an oscillatory signal that is out of phase over epochs will lead to a cancellation of the oscillatory activity. However, a more elegant way of quantifying phase resetting has been used by Rizzuto et al. (2001). For each single trial these authors extracted the instantaneous phase by convolving the raw signal with a Morlet wavelet, and taking the angle of the resulting complex coefficients. The distribution of the phase angles over trials can then be visualized by plotting it on a unit circle, and the uniformity of the distribution can be statistically evaluated, e.g. with the Rayleigh statistic (Fisher, 1993).

The second way to look at phase information is to focus on the phase relationships between two (or more) EEG signals, where the phase relation to the stimulus is of lesser importance. Such an approach can be used to identify which 
recording sites are activated synchronously, and thus may yield topographical information about the underlying cell assembly. The most widely used method to study such phenomena is the computation of event-related coherence functions (e.g. Gray et al., 1989; Roelfsema et al., 1997; see Nunez et al., 1997 for methodological considerations). Essentially, coherence is a measure of spectral covariance, which is quantified as the cross-spectrum of two signals normalized by the two autospectra (e.g., Andrew and Pfurtscheller, 1996). However, since coherence is a measure of spectral covariance, it does not separate the effects of amplitude and phase in the interrelations between two signals (Lachaux et al., 1999, 2000). This may be somewhat of a drawback for coherence analysis, since the relative contributions of phase and amplitude covariance on the coherence function are not clear. Because a consistency in the phase relation between two signals is in principle sufficient to conclude that two brain regions interact, Lachaux et al. (1999) have proposed a method termed Phase-Locking Statistics (PLS), which uniquely quantifies the phase coherence between two signals. Essentially, the method involves bandpass filtering, extracting the instantaneous phase by convolving the raw signal with a wavelet, and applying a bootstrap-like statistical test to the data (see Lachaux et al., 1999, for details).

\subsection{In Summary}

In summary, we have argued that there are crucial differences in the neurophysiological mechanisms underlying evoked and induced activity. Since induced activity is thought to reflect functional changes in the parameters controlling dynamic interactions within and between brain structures (Pfurtscheller and Lopes da Silva, 1999), we have proposed that studying this type of EEG activity may yield information about the processes involved in cell assembly formation. According to this proposal, the processes of the formation, the activation, and the cancellation of functional neuronal networks is expressed in, respectively, increases in phase consistency, increases in spectral power, and phase scattering followed by a return to baseline power. Finally, we have outlined the appropriate analytic tools for quantifying such processes. We now turn to the neural generator mechanisms that underlie different rhythms, such as alpha, theta and beta/gamma rhythms.

\section{EEG RhythMS IN THE HUMAN BRAIN}

The distinction between evoked and induced activity is valid for EEG rhythms in the entire span of frequencies contained in the human EEG (in most cases, this is from 0 to $70 \mathrm{~Hz}$ or even higher). Therefore, this paper could very well deal with rhythmic activity ranging from slow delta to the highest gamma frequencies. However, since here we are concerned with the relationship between induced activity and memory processes, we will especially focus on theta activity. As we will show, there is a clear relationship between a whole range of memory processes on the one hand, and rhythmic activity in the theta 
frequency range (roughly, 4-7 Hz) on the other hand. In order for the reader to understand the neural dynamics and functional significance of rhythmic activities in the different frequency bands, we provide a brief discussion of the alpha and beta/gamma rhythms as well.

\subsection{The Alpha and Beta/Gamma Rhythms}

\subsubsection{Alpha Rhythms}

The alpha rhythm can be defined as spontaneous waxing and waning waves within the frequency range of $8-12 \mathrm{~Hz}$, that are most pronounced during relaxed wakefulness, particularly when the eyes are closed, and that show the largest amplitudes over the occipito-parietal cortex. Sometimes a distinction is made between the upper $(10-12 \mathrm{~Hz})$ and lower $(8-10 \mathrm{~Hz})$ alpha bands. This distinction is based on differences in reactivity between these two frequency bands (see e.g. Klimesch, 1999). Despite the presence of this functional difference, research into the neural basis of alpha rhythms has not revealed a neural substrate of this distinction (see below). It should be noted that 'the' alpha rhythm is most probably not a unitary phenomenon. Even within a relatively confined cortical area such as the occipital cortex there exist a variety of rhythms, possibly reflecting activity of different thalamo-cortical circuits (e.g. Mulholland, 1969). There are good reasons to believe that alpha rhythms can be grouped on the basis of their reactivity to experimental manipulations (see, for example, the proposals in Bastiaansen et al., 1999). However, although there are obviously a large number of functionally different, parallel thalamo-cortical circuits that generate alpha rhythms, the neural mechanisms involved in the generation of different alpha rhythms are probably the same. It, therefore, makes sense to discuss the common neural basis of alpha rhythms as if they were a unitary phenomenon.

Alpha rhythms (or rather, sleep spindles, which have been used as an experimental animal model of alpha) have been most intimately linked to activity in thalamo-cortical circuits (e.g., Steriade et al., 1990; Lopes da Silva, 1991a). More specifically, alpha rhythms are thought to originate from an interplay between the thalamus and the Reticular Nucleus (RN), a thin sheet of cells surrounding the lateral aspect of the thalamus. Thalamic cells have intrinsic membrane conduction properties that lead them to operate in either of two modes: in a burst mode, which occurs when thalamic cells are hyperpolarized, and in a tonic mode when depolarized. Burst mode firing involves high-frequency discharges that recur every $100 \mathrm{~ms}$, thereby generating rhythmic activity of roughly $10 \mathrm{~Hz}$, which is then sustained through a feedback circuit involving the RN and thalamus on the one hand, and the neocortex on the other hand (for detailed reviews, see Steriade et al., 1990; Lopes da Silva, 1991a). Tonic mode firing is expressed in the absence of $10 \mathrm{~Hz}$ oscillations at the scalp, while functionally it is associated with the transmission of information from sensory afferents through the thalamus to the cortex. Burst mode firing, on the other hand, is expressed at the cortical level as $10 \mathrm{~Hz}$ oscillations (i.e., an alpha rhythm), and is functionally associated with a disruption of the transmission of information from sensory afferents through 
the thalamus to the cortex (Guillery et al., 1998). Therefore, the presence of alpha activity at the scalp is generally thought to reflect cortical idling (sensory information cannot reach the cortex), whereas its absence is thought to indicate active cortical processing of sensory (or motor) information (e.g. Pfurtscheller, 1992).

It is important to note here that the functional interpretation of event-related power changes in the alpha band is exactly opposite to that in other frequency bands: while a power increase in the alpha band is associated with decreased activity in thalamo-cortical circuits, in other frequency bands power increases reflect increases in activity. For a detailed review of the functional significance of event-related power changes in the alpha band, see Bastiaansen (2000).

\subsubsection{Beta/Gamma Rhythms}

Rhythmic EEG activity in the high frequency range $(14-30 \mathrm{~Hz})$ is usually called beta activity, while rhythms with even higher frequencies $(30-80 \mathrm{~Hz})$ are mostly referred to as gamma activity (Bressler, 1990). It should be noted that some authors do not make the distinction between beta and gamma activity (e.g. Steriade, 1997).

Despite many unknowns, knowledge about the generator mechanisms of beta/gamma rhythms is increasing. Whereas $10-\mathrm{Hz}$ activity is evident both in the cortex and thalamus, beta and gamma rhythms have been mostly associatated with the neocortex (e.g. Barth and McDonald, 1996), and with the hippocampus (e.g. Wang and Buzsaki, 1996). Inhibitory interneurons, both in the neocortex and in the hippocampal dentate gyrus, are thought to play a role in generating beta/gamma activity (Whittington et al., 2001; Bragin et al., 1995). It has been suggested that hippocampal gamma oscillations emerge from an interaction between intrinsic oscillatory properties of interneurons and the network properties of the dentate gyrus (Bragin et al., 1995). The neurotransmitter GABA has also been tightly linked to beta/gamma oscillations (Wang and Buzaki, 1996).

The role of beta/gamma oscillations in cognitive processing is a matter of debate. In general, beta and gamma rhythms are associated with behavioral conditions in which the individual is alert and focussing attention. The gamma rhythm, in particular, has been associated with processes such as feature binding (Gray and Singer, 1989; Tallon-Baudry et al., 1998), object representation (Bertrand and Tallon-Baudry, 2000), and visuomotor integration (Roelfsema et al., 1997). Underlying these functional accounts is the general idea that gamma oscillations play an important role in synchronizing activity in cortical networks, be it locally (e.g. Von Stein and Sarnthein, 2000) or between distant brain areas (Roelfsema et al., 1997).

\subsection{Theta Rhythms}

\subsubsection{Generating Brain Structures}

The theta rhythm is an EEG rhythm with a frequency between 4-7 Hz. It dominates the electrical activity recorded from the hippocampus in most mammals, but it is most prominent in rodents. Because in lower mammals there 
is considerable interspecies variation in the exact theta frequency (with frequencies ranging from $3-4 \mathrm{~Hz}$ up to $10-12 \mathrm{~Hz}$ ), the phenomenon is often termed rhythmic slow activity (RSA). Although there has been considerable debate as to whether hippocampal theta activity occurs exclusively in lower mammals or also in humans (e.g. Steward and Fox, 1990; Lopes da Silva, 1991b; Miller, 1991), it has been demonstrated, with the help of appropriate spectral analysis techniques, that the human hippocampus is able to generate theta rhythms (e.g. Halgren et al., 1978, 1985; Arnolds et al., 1980; Lopes da Silva, 1991b, 1992). A possible explanation for the relative difficulty in recording theta activity from the human hippocampus, is that its amplitude becomes more reduced and its pattern more irregular in higher mammals (Lopes da Silva, 1992).

Although the theta rhythm is commonly associated with the hippocampus, rhythmic activity with the same characteristics has also been reported for the entorhinal and cingular cortex, and for the septum (Petsche et al., 1962). There is now a fairly wide agreement that an intact septum is a necessary condition for the occurrence of the hippocampal theta rhythm, while destruction of the medial septum results in a disappearance of theta from the hippocampus and other limbic cortical areas (Petsche et al., 1962; Vinogradova et al., 1980). This strongly suggests that the septum serves as a pacemaker for the occurrence of theta rhythm in other structures. Nevertheless, Konopacki et al. (1988) have shown that hippocampal networks are capable of producing theta activity when isolated from septal input.

With respect to the hippocampal theta-rhythm, it has been established that it is not primarily determined by intrinsic membrane conductance properties, but rather by local synaptic interactions within the hippocampal formation, and parahippocampal regions such as the peri- and entorhinal cortices, and the parahippocampal (or postrhinal) cortex ${ }^{1}$ (for reviews see e.g. Steriade et al., 1990; Lopes da Silva, 1992). Apart from the hippocampal system, theta activity has been recorded in various subcortical structures, such as the cingulate gyrus (Leung and Borst, 1987), hypothalamus (Slawinska and Kasicki, 1995), and superior colliculi (Routtenberg and Taub, 1973).

More recently, theta activity has been reported for neocortical areas as well, for example, by Kahana and colleagues who used intracranial EEG measurements (e.g. Kahana et al., 1999, Caplan et al., 2001). Moreover, as we will see in section 3, spectral analyses of scalp-recorded EEG (which is dominated by cortical activity) show clear patterns of theta (re)activity in memory paradigms. However, one should realize that the occurrence of theta activity in these areas does not necessarily imply that theta is also generated in these areas.

An important, but not completely resolved issue is where the generators of the scalp-recorded theta activity are located (see section 3). One possibility is

\footnotetext{
${ }^{1}$ For the sake of clarity, we will follow the nomenclature used e.g. by Witter et al. (2000) in the remainder of this paper. Thus, we will denote the entire complex of structures mentioned here as the hippocampal system. Within the hippocampal system we will differentiate between (1) the hippocampal formation, consisting of the dentate gyrus, the fields CA1-3, and the subiculum and (2) the parahippocampal region, which includes the peri- and entorhinal cortex, and the postrhinal cortex (in nonprimates) or parahippocampal cortex (in primates including humans).
} 
that theta reflects an interplay between the hippocampal and cortical generators. It is also conceivable that there are theta generators in the neocortex itself, which may or may not act independently of the hippocampal system.

In summary, it is highly likely that a number of structures in the human brain are showing or even generating theta rhythms. Despite uncertainty about all the areas contributing or the areas necessary for it to occur, there is fairly wide agreement that the hippocampal system is involved, if not critically so. Under the assumption that modulations of theta rhythm is functionally related to the role of the hippocampus, we will now focus on the role of this system in subserving memory. However, it should be noted that it is beyond the scope of this paper to give an extensive review of the current understanding of the different functions subserved by different parts of the hippocampal system. For reviews, the reader is referred to Eichenbaum et al. (1994) or Witter et al. (2000). Instead, we will briefly describe some research findings that have linked subcomponents of the hippocampal system to different types of memory operations.

\subsubsection{Linking Structure to Function}

The hippocampal system, in intimate relation with neocortical association areas, contributes critically to learning and memory. The current understanding of the cortico-parahippocampal-hippocampal network seems to support a number of functionally dissociable components of the hippocampal system (cf. Witter et al., 2000). For example, the perirhinal cortex appears to be involved in object recognition memory (Meunier et al., 1993). Spatial memory appears to be mediated by the postrhinal cortex (in nonprimates) or the parahippocampal cortex (in monkeys; e.g. Maguire et al., 1996; Malkova and Mishkin, 1997). The parahippocampal cortex has recently been related to successful retrieval operations (Daselaar et al., 2001). A more general proposal, by Eichenbaum and colleagues (Eichenbaum et al., 1994, 1996) is that two different functions are mediated by the hippocampal system. While the parahippocampal region supports intermediate-term storage of individual items, the hippocampal formation is proposed to play a role in mediating relationships between elements of a particular episode.

Obviously, the hippocampal system is not solely responsible for all the aspects of human memory performance, including short-term, intermediate-term and long-term storage. Eichenbaum et al. (1994, 1996), amongst others, have proposed that neocortical association areas play an important role in storing memories. In their view, neocortical association areas maintain short-term memories for specific items prior to hippocampal processing, and eventually provide the final repositories of long-term memory. The latter idea is consistent with that proposed by Miller (1991), that "the hippocampus (...) acts in some ways as an 'index' to memories, the cortex being, as it were, the 'book' to which this index refers" (Miller, 1991, p. 159). Similar, although slightly different ideas have been put forward by Treves and Rolls (1994). These authors hypothesize that the hippocampal formation (more specifically, the CA3 region), operates as a single autoassociation network that stores new episodic information 
as it arrives from many cortical association areas. The CA3 system could then be used to recall a whole episode when only a fragment of it is presented. This would be accomplished by reinstating neuronal activity in the association areas of the neocortex, activity that is similar to the activity that was present during the original episode.

The anatomical framework for the conception of cortico-hippocampal interplay in memory performance is that the parahippocampal region mediates the reciprocal transfer of information between a variety of multimodal association cortices and the hippocampal formation (Lopes da Silva, Witter, Boeijinga et al., 1990). Thus, in order to perform the proposed functions, the different structures of the hippocampal system have to interact prominently with neocortical association areas. It has been proposed (Miller, 1991) that this transfer of information between the hippocampal system and cortical association areas may well be mediated by rhythmic activity in the theta frequency range. We will discuss this proposal in some detail below.

Miller (1991) proposes that Hebbian processes of synaptic strengthening select patterns of recursive loops passing from the hippocampal formation through the parahippocampal region to the cortex, and back again. Based on average estimates of axonal conduction times in the rat, he arrives at a total conduction time for one loop of 120 to $200 \mathrm{~ms}$, which roughly corresponds to one period of theta. Under these conditions, the Hebbian mechanism favors the strengthening of connections that permit resonance. Thus, patterns of resonant loops emerge between the cortex and the hippocampal system, which are strengthened by the precise timing (at frequencies corresponding to the theta range) of cell firing at the hippocampal end of the loop. In Miller's view, this process of self-organization of cortico-hippocampal loops has an important functional role both in the storage and in the retrieval of cortically represented memories. One of the major functions of this cortico-hippocampal interplay is that each pattern of loops (corresponding, say, to one single episode) will synchronously activate functionally related, but widely dispersed cells in the neocortex, which by themselves have insufficient mutual interconnections to be able to form a cell assembly by the usual Hebbian principles.

In summary then, Miller proposes that cortico-hippocampal interactions at theta frequencies may serve the goal of establishing long-range cortico-cortical synchronization of functionally related cells, which would not show synchronization without the orchestrating role of the hippocampal system. Note that this idea is consistent with the observation of Kahana and coworkers (e.g. Kahana et al., 1999; Caplan et al., 2001) that theta recorded directly from the cortical surface varies considerably both in amplitude and in phase across distances of only 1-2 cm. A spatially distributed cell assembly, activated via the hippocampal system, is compatible with such findings. It is interesting to note that Von Stein and Sarnthein (2000) have also proposed that theta subserves the goal of integrating neural activity in distant cortical areas (e.g. between frontal and parietal cortices). Although it is unclear whether they conceive of such longrange cortico-cortical integration as being entirely cortical in nature, without mediation by the hippocampal system, their proposal is not inconsistent with the cortico-hippocampal interplay at theta frequencies as proposed by Miller (1991). 
An important implication of Miller's (1991) notion of self-organizing, resonant loops between the hippocampal system and the neocortex, is that studying the reactivity of theta at the cortical end of the loop (either intracranially or with scalp EEG) might provide us with a window on the hippocampal contribution to different types of memory processes. The major advantage of such an approach is that it would be possible to use scalp recordings for investigating (at least globally) hippocampal function. However, such an approach would assume that all of the theta activities recorded at the scalp originate in cortico-hippocampal circuits. As we have seen above, this is as yet unclear. Empirical studies combining intracranial recordings in the hippocampal system with scalp recordings are highly desirable, since they could directly address the question of the relationship between hippocampal theta and scalp-recorded theta by using the analysis tools described in section 1.2.

\section{The Reactivity of (Cortical) Theta in Memory Paradigms}

The literature review presented in this section focuses on experimental work in which the reactivity of theta rhythms in the human EEG (recorded at the scalp or in the cortex) has been studied in memory paradigms. We present this review here because we reason that studies demonstrating clear relations between the reactivity of scalp- (or intracranially) recorded theta during memory paradigms can, in principle, provide evidence for the proposal that induced theta rhythms play a functional role in cell assembly formation related to mnemonic processing. In addition, such studies may provide supporting evidence in favor of the hypothesis that communication between the hippocampal system and the neocortex takes place at theta frequencies. With respect to the latter, it should be emphasized again that such evidence can only be highly indirect, since it is unlikely that all of the theta activity at the cortex is generated in the hippocampal system, as we pointed out in the previous paragraph.

In the last subsection, we discuss studies that have addressed theta reactivity in relation to language processing, and we argue that these studies address processes that are at the interface between memory and language. In the overview, we give a more detailed description of some of our own work.

\subsection{Theta and Encoding/Retrieval of Information}

Klimesch and coworkers studied the event-related changes in scalp-recorded theta power during the performance of episodic and semantic memory tasks using IBP analysis (outlined in section 1.2.1). Typically, in those studies, subjects read a word list, or had to perform a semantic judgement task. Subsequently they were asked to perform either a free or a cued recall task (see Klimesch, 1996; 1999 for reviews). These experiments provided convincing evidence for an event-related increase in theta power during both the encoding and the retrieval of episodic information, which is larger for good than for poor memory performers (e.g. Klimesch et al., 1990; Klimesch et al., 1996; Klimesch 
et al., 1997a). For example, Klimesch et al. (1997b) asked subjects to judge the semantic congruency of feature-concept pairs (such as "claws - eagle"). After that, an episodic memory task was performed, in which subjects had to recall the concept word that was actually paired with a feature word in the initial list. On the basis of recall scores, subjects were divided in a high-performance and a low-performance group. Contrasting the theta power changes for words that were later recalled vs. not recalled, the authors found that only recalled words were associated with a significant theta power increase. Moreover, this effect was only present for the high memory performers.

In additional studies, it was shown that event-related increases in theta power are generally larger during retrieval than during encoding (e.g. Klimesch et al., 1997c; Klimesch et al., 1994). For example, in a recent study (Klimesch et al., 2001), in which pictures were used rather than words, subjects viewed 70 pictures in the study phase of the experiment. After about six minutes, subjects were asked to make a recognition judgement for 180 pictures, i.e. 60 previously seen pictures (targets) and 120 distracters. Whereas baseline (i.e., pre-stimulus) levels of theta power were similar during the study phase and the test phase prior to the presentation of both targets and distracters, post-stimulus theta power increased by approximately 50\% (relative to pre-stimulus theta power) during the study phase (encoding) of the experiment. During the test phase (retrieval), theta power increases relative to the pre-stimulus interval were in the range of $100-150 \%$ for correctly identified targets and distracters. An increase in theta power during memory retrieval has also been observed by Burgess and Gruzelier $(1997,2000)$ during the recognition of both words and faces.

The notion of a theta power increase during both encoding and retrieval is in accordance with a cell assembly formation hypothesis, since, as we argued (see section 1.1), activation of a cell assembly is expected to lead to a power increase in the relevant frequency band. Unfortunately, we have not been able to find studies investigating changes in theta phase in episodic memory tasks. Such studies are important because they could provide a more complete picture of all aspects of theta reactivity during semantic or episodic memory encoding and retrieval.

\subsection{Theta and Working Memory}

Studies addressing the relation between theta activity and working memory (WM) have focused either on changes in theta power, or on changes in theta phase. We first discuss studies addressing power changes in relation to working memory. Gevins et al. (1997) used a visual n-back task, manipulating stimulus content (verbal vs. spatial) and WM-load. They assessed task-related changes in theta power in the concurrently measured scalp EEG. Both for the verbal and for the spatial WM task, Gevins et al. found an increase in theta power at frontal midline electrodes, that was larger for conditions with higher memory load. Furthermore, theta power increased with practice, and more so in the high than in the low WM-load conditions. In a subsequent study (McEvoy et al., 2001), the authors further demonstrated that the increase in frontal midline theta with 
increased WM-load was present only in young subjects, not in older ones. The authors concluded that their data confirm the existence of a fronto-parietal network involved in WM.

Another study relating theta to WM was recently reported by Krause et al. (2000). These authors studied event-related power changes in a verbal n-back task. Although specific effects of memory load were present only in higher (that is, 6-8 and 8-10 Hz ) frequency bands, the results did show a widely distributed increase in theta power in the first $500 \mathrm{~ms}$ following each new item, that is, in the interval in which WM processes were assumed to be active. The authors related this effect to either stimulus identification or working memory processes. In a subsequent experiment, Krause et al. (2001) investigated the test-retest reliability of the WM-related theta response using a Sternberg task. In this task, subjects heard a set of four words, followed by a probe word three seconds later. Subjects were required to indicate whether or not the probe had been present in the memory set. Two sessions were performed, at least nine days apart. Again, a theta increase was found during the retention interval of the task. Additionally, the test-retest reliability was largest for the theta band, while concurrently examined higher frequency bands (up to $12 \mathrm{~Hz}$ ) showed large significant differences between the two sessions.

In the studies by Krause et al., verbal material was employed in the working memory tasks. In order to establish the relation between event-related power changes and spatial WM, we performed a large study (Bastiaansen et al., 2002a) using a human adaptation of the classical Delayed Response Task (DRT), which is often used in animal research (see Geffen et al., 1997 for a review). Subjects had to fixate on a central spot on a touch-sensitive computer monitor. A target was presented in the periphery. The target location had to be remembered. After a delay (either $1 \mathrm{~s}$ or $4 \mathrm{~s}$, in order to manipulate the WM-load), the fixation stimulus disappeared, and subjects had to respond by touching the screen at the location of the target. 'Sensory' trials, in which the target remained visible on the screen, were randomly intermixed with 'memory' trials, in which the target disappeared $150 \mathrm{~ms}$ after its appearance. Thus, the sensory trials form a control condition in which the response demands are highly similar to those in the memory trials. A large number of subjects were used for the study $(\mathrm{N}=174)$. The results of this study with respect to the theta band are presented in Figure 1.

In the first $500 \mathrm{~ms}$ after presentation of the target stimulus a phasic increase in theta power was found, irrespective of condition and delay interval, in the lower (roughly, 4-7 Hz) frequency bands with a maximum over parietal areas. This effect was interpreted to reflect activity in a hippocampal-parietal loop related to the encoding of the location of the target stimulus. Most surprisingly, we found that both the sensory and the memory trials showed a power decrease in the theta band over the (lateral) frontal electrodes irrespective of condition. Although this effect may be attributed to task execution, it was much larger in the memory compared to the sensory trials. Note that Figure 1 reveals that IBP differences between the memory and sensory trials occur 100-150 ms before the point in time where the two trial types start to diverge. However, this is 


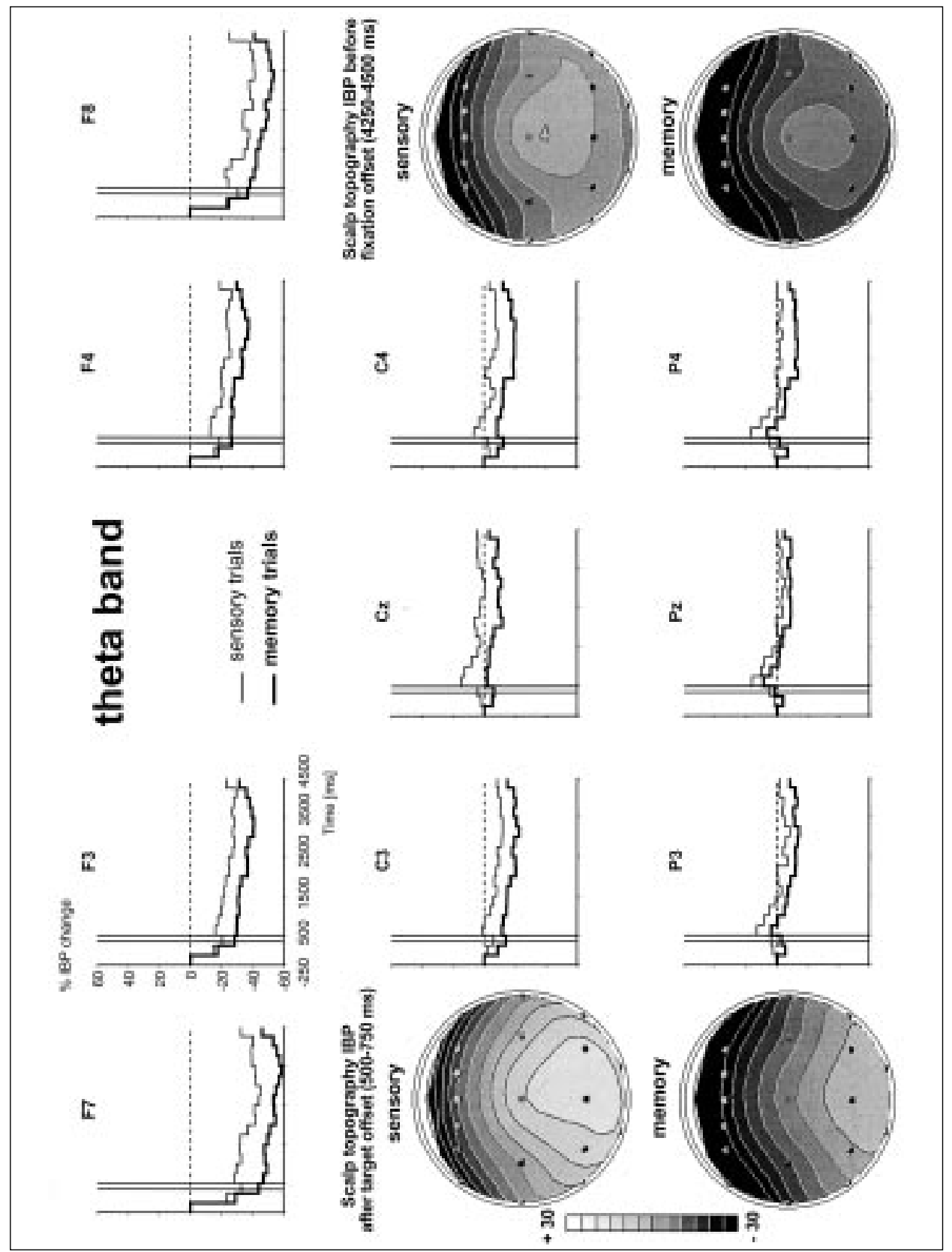

Fig. 1 - Grand average $(N=174)$ time courses at selected electrodes, and topographic maps at selected time points, of the Induced Band Power (IBP) changes in the theta frequency band in the sensory and memory conditions. IBP changes are shown from $250 \mathrm{~ms}$ preceding trial onset to the end of the memory delay interval. $t=0$ corresponds to the onset of the fixation point, the target appears $350 \mathrm{~ms}$ later. For the time course plots, vertical lines indicate onset and offset of target stimulus (offset only for memory conditions). For the topographical maps, power decrease is represented by dark gray surrounded by white contour lines, power increase by light gray surrounded by black contour lines. Grey contour lines correspond to zero power change. Spacing of contour lines is $5 \%$ IBP change. 
probably an artifact caused by the poor temporal resolution of IBP measures, as we noted in section 1.2.1 (see also Knosche and Bastiaansen, 2002). Hence, spatial working memory in this experiment was accompanied by an event-related decrease in theta power at frontal electrodes.

It is difficult to reconcile these findings with the well-known involvement of dorso-lateral prefrontal cortex in WM (see Bastiaansen, et al., 2002a for a discussion). If one is willing to assume that scalp-recorded activity in the theta frequency range is generated by (para)hippocampo-cortical circuits, then the logical consequence would be that the power decrease is a reflection of a reduced activity in these circuits. A speculative interpretation of such reduced activity is to ascribe it a functional role in enhancing the signal-to-noise ratio of the activity in the hippocampo-parietal loop involved in encoding (and possibly maintaining) the location of the target stimulus, by reducing the noise (i.e. the activity in other hippocampo-cortical loops) relative to the signal (i.e. the activity in the hippocampo-parietal loop). An argument in favor of such an interpretation is that, irrespective of condition, the scalp topography of the IBP in the theta band showed maximal power at parietal electrodes throughout the entire WM interval (see topographical maps in Figure 1), whereas the main difference between sensory and memory conditions is the power reduction at other scalp locations.

Up to now, two studies have addressed event-related changes in theta phase during WM tasks. In a recent study by Kahana and coworkers (Rizzuto et al., 2001), EEG was recorded subdurally, with chronically implanted electrodes in patients undergoing evaluation for surgical treatment of epilepsy. Subjects performed a Sternberg task, in which four consonants were presented, followed by a probe item. They were asked to indicate whether or not the probe was a member of the previous list. During both encoding and retrieval stages, sustained theta oscillations were identified. Most interestingly, theta phase was randomly distributed preceding the probe item, whereas after the presentation of the probe (i.e., during retrieval) theta phase clustering was clearly evident in most electrodes. In addition, in some electrodes such a phase clustering was also observed following the presentations of the list items, i.e., during encoding. No clear topographical pattern was present for these effects. Rather, the pattern of reset often changed dramatically from one electrode to the neighboring one, again pointing to the existence of widely distributed yet functionally related networks. These findings indicate that memory encoding and retrieval operations produced a reset of the phase of the theta rhythm. Similar findings were obtained by Tesche and Karhu (2000), who measured MEG signals during a Sternberg task, and modeled the theta oscillations by an equivalent current dipole in the anterior hippocampus. Tesche and Karhu (2000) showed a similar pattern of phase resetting (of the activation time course of the current dipole) following probe presentations. In addition, they found that larger sets of list items led to longer periods of theta oscillations once the probe was presented, at least for the in-set probes, while theta oscillations for out-of-set probes was longer than for the in-set probes.

In summary, research using a variety of WM paradigms has shown (i) a phase resetting during the periods when items have to be stored or retrieved 
from WM, and (ii) a power increase in the first $500 \mathrm{~ms}$ after the presentation of an item on which WM operations have to be performed (note however that in the Bastiaansen et al. (2002a) study the power increase was restricted to some electrodes, while others showed a power decrease). In general, then, this pattern of results fits well with the concept of cell assembly formation proposed in section 1. It should be noted that a precise understanding of the timing of the phenomena of phase resetting and power increase is as yet lacking, but crucial for our proposal. The relatively poor temporal resolution of frequency-domain analyses of the EEG (that is, compared to ERPs), which is roughly in the order of a few hundred milliseconds, may hinder an understanding of the timing of the phenomena at the millisecond level. However, using an appropriate experimental paradigm it may be possible to establish at least the relative timing of phase resetting and power increase, which would be sufficient for the present purpose.

\subsection{Theta and Spatial Memory}

Theta oscillations can be recorded from the hippocampus of rodents when they engage in a variety of tasks. For many years, theta has been intimately related to spatial navigation (e.g. Winson, 1978). For example, theta is related to the firing of hippocampal place cells that encode the animal's position (e.g. O'Keefe and Recce, 1993). However, it is only recently that some researchers have begun studying the relation between theta activity and spatial memory in humans. Therefore the evidence relating theta at the cortical level to spatial memory is at present meager, and consists of only a few studies. Kahana et al. (1999) devised a testing procedure in which subjects have to navigate in virtual, three-dimensional mazes, in one of two modes: A study mode, in which arrows direct subjects through the maze, and a test mode, in which the arrows are removed. Three epileptic patients with chronically implanted subdural electrodes, who were being evaluated for surgical treatment, participated in the experiment. Prominent theta activity was recorded by a large number of subdural electrodes during both modes; however, theta episodes occurred more frequently during test mode (associated with retrieval operations) than during study mode (related to encoding operations). Moreover, longer (i.e., more difficult) mazes, as compared to shorter mazes, were associated with a larger percentage of time during which theta oscillations were present, and theta bursts were of longer duration in long mazes as opposed to short mazes. In a subsequent study (Caplan et al., 2001), these results were confirmed with a larger number of subjects (i.e. five). These preliminary data indicate a clear relationship between power increases of cortical theta and spatial memory.

\subsection{Theta at the Interface between Memory and Language}

In section 2.2, we reviewed some neuroimaging studies showing that the hippocampal system contributes to different types of memory operations. However, it seems likely that the hippocampal system does not only contribute to mnemonic processes, but also to at least some aspects of language processing. Indeed, a number of recent studies have shown relationships between linguistic tasks and activity in the hippocampal system, relationships which appear to 
roughly parallel the different functions Eichenbaum et al. (1994) ascribed to parahippocampal region and hippocampal formation, that is, intermediate-term storage of individual items and organization of the relevant relationships between individual items, respectively (e.g. Daselaar et al., 2001; Crinion et al., 2001; Crosson et al., 2001; , Taylor et al., 2001; Keller et al., 2001; Gleissner and Elger, 2001).

In language processing relevant information has to be encoded in memory and to be kept available for integrating additional linguistic materials into the preceding sentence or discourse. It seems, therefore, reasonable to hypothesize a similar functional role for theta activity during particular language tasks as during memory tasks. In addition, activities in the hippocampal system during language tasks may lead to changes in theta-band activity in cortico-hippocampal loops in the same way as we argued for memory tasks. In this case, again, we would expect such activity to become manifest at the cortical end of the loop, where we might be able to detect it with scalp electrodes. Of course, the same caveats as we expressed in section 2.3 apply to this reasoning. Although until now only a few studies have been performed which address this question explicitly, they indeed appear to show such patterns of event-related reactivity in the theta band. We will discuss these studies in some detail below.

Bastiaansen et al. (2002b) investigated the event-related power changes in the alpha and theta bands with the IBP technique, while subjects read correct Dutch sentences in serial visual presentation mode (for a full discussion of the results, see Bastiaansen et al. (2002b). Analyses were performed both at the word-level (with a $300 \mathrm{~ms}$ baseline preceding each word, irrespective of its position in a sentence) and at the sentence level (with a $600 \mathrm{~ms}$ pre-sentence baseline, focusing on the evolution of power over the entire sentence).

Results of the word-level analysis, shown in Figure 2, indicate that in the theta band, a spatially widespread, phasic power increase can be identified. Averaged over electrodes and word positions, this power increase reaches a maximum at 320 ms following word onset. It should be noted that the temporal resolution of the IBP method is not as good as that of ERP measures (e.g. Clochon et al., 1996). Therefore the value of the peak latency should be taken only as a rough indication of the timing of the neurophysiological phenomena driving the IBP response. Nonetheless, this latency roughly falls in the range of well-known ERP components related to language processing, such as the N400 (Kutas and Hillyard, 1980) and the Left Anterior Negativity (LAN; e.g. Munte et al., 1997). Hence, it seems reasonable to hypothesize that the power increase in the theta band is related to some aspect of word processing, either lexical or in relation to sentence context. With the literature reviewed in section 3.1 in mind, possible candidate processes might be (i) the encoding of the word, (ii) the retrieval of word meaning, or (iii) the integration of word meaning into an overall representation of the sentence. It should be noted that in the (upper) alpha band we found a power decrease after word onset. Given that alpha has been intimately related to sensory processing (cf section 2.1.1, see also Bastiaansen and Brunia, 2001), we attributed this effect to the visual processing involved in reading the words.

Results of the sentence-level analysis, (see Figure 3), show that in the theta band, a slow, gradual increase in power can be observed as the sentence unfolds. 


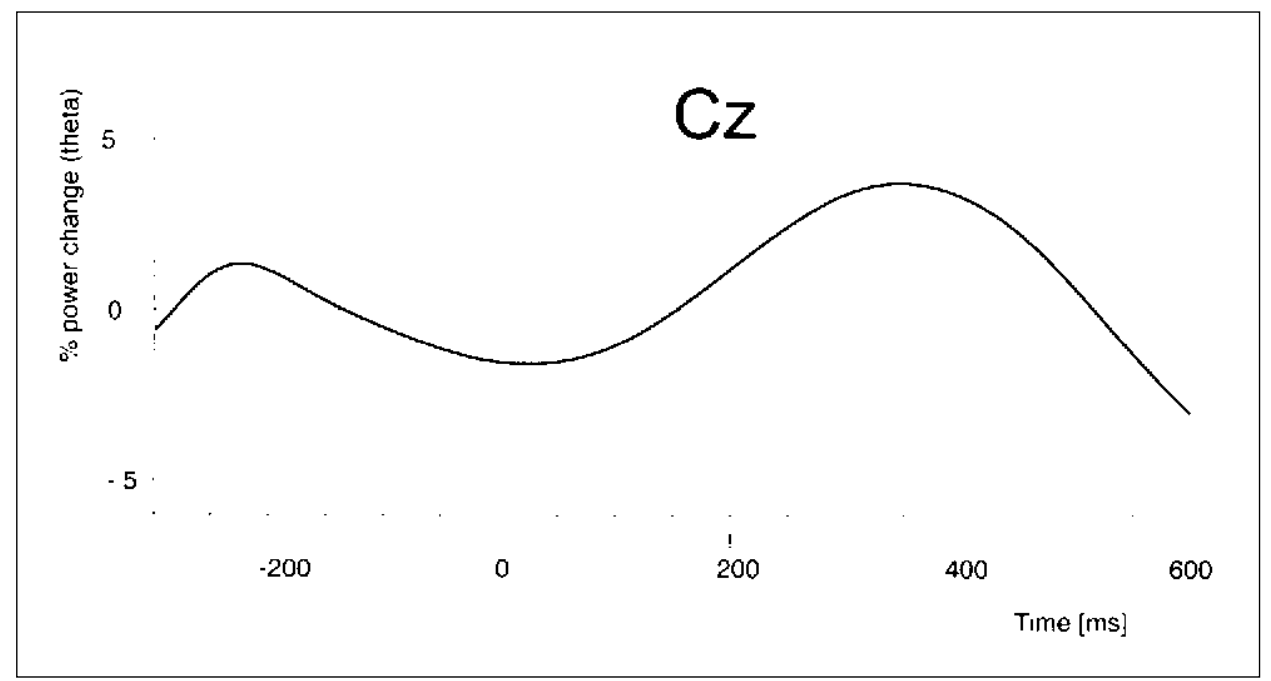

Fig. 2 - Grand average $(N=18)$ temporal evolution of IBP changes in the theta frequency band, averaged over word positions, for electrode $C z$.

Testing each and every electrode for the presence of a linear trend, we found significant trends in a cluster of electrodes located bilaterally over the temporal cortex, as well as in electrodes over the (pre)central midline ( $\mathrm{Cz}$ and $\mathrm{FCz}$ ). A tentative interpretation of the linear increase in theta power over the sentence may be that it reflects the gradual building up of a memory trace related to the encoding of the linguistic episode, with higher processing demands as each new word of the sentence is added to the memory trace. In other frequency bands we did not find any linear trends. It should be noted that the predominance of synchronization in the alpha range, apparent in Figure 2, contrasts with the usually reported combination of theta increase/alpha decrease (e.g. Klimesch, 1999; see Bastiaansen et al., 2002b for a more extensive discussion of the findings).

Weiss, Muller and Rappelsberger (2000) investigated the event-related coherence of theta activity during the encoding of auditorily presented nouns. Fifty concrete and 50 abstract nouns were presented in random order in 4 blocks of 25 nouns each. After the presentation of each block, subjects were asked to recall as many of the nouns presented in that block. Recall was significantly better for concrete than for abstract nouns. The results showed that later recalled nouns were associated with higher levels of theta coherence than nouns that were not recalled later. Most interestingly, this difference was larger for long-range coherence values (defined as coherence between non-adjacent electrodes) than for short-range coherence (i.e., between adjacent electrodes). Moreover, abstract nouns were more associated with long-range coherence than concrete nouns.

Interestingly, in both studies discussed here, the results have been interpreted mainly in terms of processes that lie at the interface between memory and language - that is, the processes of encoding, storing and/or retrieving of individual words, and the building up of a memory trace as a sentence unfolds. In our view, it has yet to be established whether the theta responses observed in 


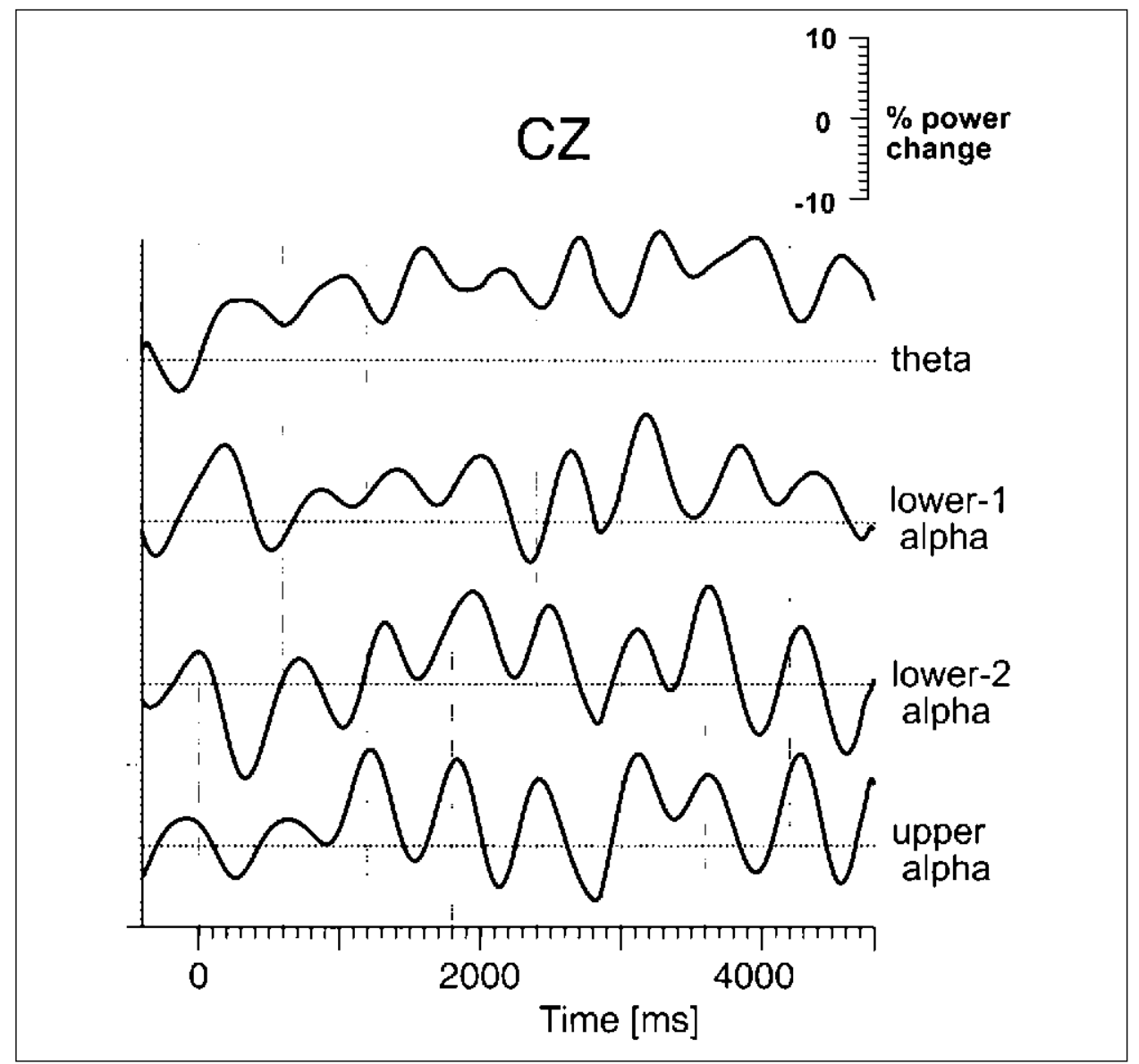

Fig. 3 - Grand average $(N=18)$ temporal evolution of IBP changes during the presentation of the first 8 words of a sentence, relative to a pre-sentence reference interval, for electrode $C z$. Note the slow IBP increase over time, which is present as a significant linear trend only in the theta band.

linguistic tasks reflect general mnemonic processes, or more domain-specific linguistic processes. Studies using more fine-grained linguistic manipulations could be helpful in finding an answer to this question.

\section{CONCLUSIONS}

In section 3 we have reviewed the literature on the reactivity of theta rhythms in humans, in a variety of memory paradigms, and during language processing. This review was presented in order to evaluate two hypotheses. First, the hypothesis that theta reactivity reflects processes of cell assembly formation related to different types of memory operations. Second, the (stronger) hypothesis that theta recorded at the cortical level may provide a window on the contribution of the hippocampal system to memory processes. 
With respect to the first hypothesis, we can conclude that the available experimental evidence speaks in favor of a relation between theta and memoryrelated cell assembly formation. Theta power increases, which we have hypothetically related to the activation of a cell assembly, are usually found during tasks which engage encoding/retrieval of episodic information, working memory, spatial memory or language processing. The few reports that are available on theta phase resetting indicate a relation between encoding/retrieval of information from working memory on the one hand, and theta phase resetting, hypothesized to reflect the actual formation of a cell assembly, on the other hand. In a similar way, an increase in theta phase coherence between spatially distant recording sites, hypothesized to yield topographical information about activated cell assemblies, has been reported in relation to language processing. However, what is needed for a more thorough evaluation of our hypothesis, is systematic research into (the relative timing of) all the relevant parameters: (i) phase resetting (cell assembly formation), (ii) power increase and inter-electrode phase coherence (cell assembly activation), and (iii) phase scattering followed by power decrease to baseline levels (uncoupling of the cell assembly). Quantifying all of these parameters, during different memory paradigms, may lead to a better understanding of the proposed relation between theta rhythms and memory-related processes of cell assembly formation.

With respect to the second hypothesis, there is a strong parallelism between the fact that the hippocampal system is both involved in certain aspects of memory and in generating theta activity, and the finding that scalp-recorded theta shows a strong reactivity in memory paradigms. Although it is tempting to conclude that these findings confirm our hypothesis, such a conclusion is highly inferential and potentially misleading. We would like to emphasize once more that the literature reviewed in section 3 can only provide highly indirect evidence in favor of the notion that theta reactivity may yield information about hippocampal contributions to mnemonic processes. As we noted earlier, direct evidence could be obtained by performing studies which combine intracranial recordings in the hippocampal system with scalp recordings. Clear and predictable relationships between phase and amplitude of hippocampal theta, and phase and amplitude of scalp-recorded theta, would constitute convincing evidence for the view that we presented.

Finally, despite many unknowns we believe that exploiting the modulations of the brain's rhythmicity in well-controlled experimental studies and with the help of the analysis tools that we described, may provide new vistas on the dynamics of the neurophysiological processes underlying human cognition. The accumulated evidence in neuroimaging studies over the last decade has made it increasingly clear that without a better understanding of the dynamics of cell assembly formation in functionally linking distant brain areas, much of the organizational principles of the neural instantiation of cognitive processes will remain mysterious. It is therefore, that we believe that the type of analyses explicated above hold promise as an additional important way to study brain function.

Acknowledgements. The authors wish to thank Pascal Fries, Mike Kahana, Wolfgang Klimesch, Peter Indefrey, and three anoymous reviewers for their useful comments on an earlier version of this paper. 


\section{REFERENCES}

ANDREw C and Pfurtscheller G. Event-related coherence as a tool for studying dynamic interaction of brain regions. Electroencephalography and Clinical Neurophysiology, 98: 144-148, 1996.

Arnolds DEAT, Lopes da SiLva FH, Aitink JW, KAMP A and BoEIJINGA P. The spectral properties of hippocampal EEG related to behavior in man. Electroencephalography and Clinical Neurophysiology, 50: 324-328, 1980

Basar E, Schurmann M, Demiralp T, Basar-Eroglu C and Ademoglu A. Event-related oscillations are 'real brain responses' - wavelet analysis and new strategies. International Journal of Psychophysiology, 39: 91-127, 2001.

BARTH DS and MACDONALD KD. Thalamic modulation of high-frequency oscillating potentials in auditory cortex. Nature, 383: 78-81, 1996.

BASTIAANSEN MCM. Anticipatory attention: An event-related desynchronization approach. Ph. D thesis. Tilburg University, the Netherlands, 2000.

BASTIAANSEN MCM and BRUNIA CHM. Anticipatory attention: An event-related desynchronization approach. International Journal of Psychophysiology, 43: 91-107, 2001.

BASTIAANSEN MCM, BRUNIA CHM and BÖCKER KBE. ERD as an index of anticipatory behavior. In G Pfurtscheller, F Lopez da Silva (Eds), Event-Related Desynchronization. Handbook of Electroencephalography and Clinical Neurophysiology, revised series, Vol 6. Amsterdam: Elsevier, 1999, pp. 203-217.

Bastiannsen MCM, Posthuma D, Groot PFC and DE Geus EJC. Event-related theta responses in a visuo-spatial working memory task. Submitted, 2002a.

BastiaAnSEN MCM, VAN BERKUM JJA and HAgOORT P. Event-related theta responses in the human EEG during online sentence processing. Neuroscience Letters, 324: 121-124, 2002b.

BERGER H. Über das Elektroencephalogramm des Menschen. Archiv fur Psychiatrische NervenKrankheit, 87: 527-570, 1929.

Bertrand O and TALlON-BAUDRY C. Oscillatory gamma activity in humans: A possible role for object representation. International Journal of Psychophysiology, 38: 211-223, 2000.

Bragin A, Jando G, Nadasdy Z, HetKe J, Wise K and Buzsaki G. Gamma (40-100 Hz) oscillation in the hippocampus of the behaving rat. Journal of Neuroscience, 15: 47-60, 1995.

BRESSLER S. The gamma wave: A cortical information carrier? Trends in Neuroscience, 13: 161-162, 1990.

BuLLOCK TH. Introduction to induced rhythms: A widespread, heterogeneous class of oscillations. In E Basar and TH Bullock (Eds), Induced rhythms in the brain. Boston: Birkhauser, 1992, pp. $1-26$.

Burgess AP and GruZelier JH. Short duration synchronization of human theta rhythm during recognition memory. Neuroreport, 8: 1039-1042, 1997.

BuRgESS AP and GRUZELIER JH. Short duration power changes in the EEG during recognition memory for words and faces. Psychophysiology, 37: 596-606, 2000.

Caplan JB, Madsen JR, Raghavachari S and Kahana MJ. Distinct patterns of brain oscillations underlie two basic parameters of human maze learning. Journal of Neurophysiology, 86: 368-380, 2001.

Clochon P, Fontbonne JM, Lebrun N and Etévenon P. A new method for quantifying EEG eventrelated desynchronization: Amplitude envelope analysis. Electroencephalography and Clinical Neurophysiology, 98: 126-129, 1996.

CRINION J, BLANK SC and WiSE R. Central neural systems for both narrative speech comprehension and propositional speech production. NeuroImage, 13: S520, 2001.

Crosson B, Benefield H, Cato MA, Sadek JR, Gopinath K, Soltysik D, Bauer RM, Auerbach EJ, GOKCAY D, LEONARD CM and BRIGGS RW. Left parahippocampal gyrus activity during language generation. NeuroImage, 13: S521, 2001.

Daselaar SM, Rombouts SARB, Veltman DJ, RaAijmakers JGW, Lazeron RHC and Jonker C. Parahippocampal activation during successful recognition of words: A self-paced event-related fMRI study. NeuroImage, 13: 1113-1120, 2001.

Eichenbaum H, OtTo T and Cohen NJ. Two functional components of the hippocampal memory system. Behavioural and Brain Science, 17: 449-517, 1994.

Eichenbaum H, Schoenbaum G, Young B and Bunsey M. Functional organization of the hippocampal memory system. Proceedings of the National Academy of Sciences USA, 93: 13500-13507, 1996.

FISHER N. Statistical analysis of circular data. Cambridge, England: Cambridge University Press, 1993.

FRISTON KJ, STEPHAN KM and FRACKOWIAK RJS. Transient phase-locking and dynamic correlations: Are they the same thing? Human Brain Mapping, 5: 48-57, 1997.

GefFen TM, Wright MJ, GreEn HJ, Gillespie NA, Smyth DC, Evans DM and GefFen LB. Effects of memory load and distraction on performance and event-related slow potentials in a visuo-spatial working memory task. Journal of Cognitive Neuroscience, 9: 743-757, 1997.

Gevins A, Smith ME, McEvoy L and Yu D. High-resolution EEG mapping of cortical activation related 
to working memory: Effects of task difficulty, type of processing, and practise. Cerebral Cortex, 7: 374-385, 1997.

GLEISSNER U and Elger CE. The hippocampal contribution to verbal fluency in patients with temporal lobe epilepsy. Cortex, 37: 55-63, 2001.

Gray CM, Konig P, ENGEL AK and Singer W. Oscillatory responses in cat visual cortex exhibit intercolumnar synchronization which reflects global stimulus properties. Nature, 338: 334, 1989.

GrAY CM and SINGER W. Stimulus-specific neuronal oscillations in orientation columns of cat visual cortex. Proceedings of the National Academy of Sciences USA, 86: 1698-1702, 1989.

Guillery RW, Feig SL and Loszadi DA. Paying attention to the thalamic reticular nucleus. Trends in Neuroscience, 21: 28-32, 1998.

Halgren E, BABB TL and CRANDAll PH. Human hippocampal formation EEG desynchronizes during attentiveness and movement. Electroencephalography and Clinical Neurophysiology, 44: 778-781, 1978.

HalgRen E, Smith ME and Stapleton JM. Hippocampal field-potentials evoked by repeated vs. nonrepeated words. In G Buszaki and $\mathrm{CH}$ Vanderwolf (Eds), Electrical Activity of the Archicortex. Akademiai Kiadó, Budapest, 1985, pp. 67-81.

Kahana MJ, SeKuler R, CAPlan JB, Kirschen M and Madsen JR. Human theta oscillations exhibit task dependence during virtual maze navigation. Nature, 399: 781-784, 1999.

KAlChER J and PfurTSCHELlER G. Discrimination between phase-locked and non-phase-locked eventrelated EEG activity. Electroencephalography and Clinical Neurophysiology, 94: 381-384, 1995.

Keller S, Downes JJ, Mackay C, Westwell N, Baker G and Roberts N. Language ability and hippocampal integrity. NeuroImage, 13: S549, 2001.

KLIMESCH W. Memory processes, brain oscillations and EEG synchronization. International Journal of Psychophysiology, 24: 61-100, 1996.

KLIMESCH W. EEG alpha and theta oscillations reflect cognitive and memory performance: A review and analysis. Brain Research Reviews, 29: 169-195, 1999

Klimesch W, Doppelmayr M, Schwaiger J, Winkler T and Gruber W. Theta oscillations and the ERP old/new effect: Independent phenomena? Clinical Neurophysiology, 111: 781-793, 2000.

Klimesch W, Doppelmayr M, Russeger H and Pachinger T. Theta band power in the human scalp EEG and the encoding of new information. Neuroreport, 7: 1235-1240, 1996.

Klimesch W, Doppelmayr M, Pachinger T and Russeger H. Event-related desynchronization in the alpha band and the processing of semantic information. Cognitive Brain Research, 6: 83-94, 1997a.

Klimesch W, Doppelmayr M., PAChinger T and RipPer B. Brain oscillations and human memory performance: EEG correlates in the upper alpha and theta bands. Neuroscience Letters, 238: 9-12, $1997 b$.

Klimesch W, Doppelmayr M, Schimke H and Ripper B. Theta synchronization in a memory task. Psychophysiology, 34: 169-176, 1997c.

Klimesch W, Doppelmayr M, Stadler W, Pollhuber D, Sauseng P and Rohm D. Episodic retrieval is reflected by a process-specific increase in human electroencephalographic theta activity. Neuroscience Letters, 302: 49-52, 2001.

Klimesch W, Schimke H, Ladurner G and Pfurtscheller G. Alpha frequency and memory performance. Journal of Psychophysiology, 4: 381-390, 1990.

Klimesch W, Schimke H and Schwaiger J. Episodic and semantic memory: An analysis in the EEG theta and alpha band. Electroencephalography and Clinical Neurophysiology, 91: 428-441, 1994.

Klimesch W, Russegger H, Doppelmayr M and Pachinger TH. A method for the calculation of induced band power: Implications for the significance of brain oscillations. Electroencephalography and Clinical Neurophysiology, 108: 123-130, 1998.

KNOSCHE TR and BASTIAANSEN MCM. On the time resolution of event-related desynchronization: A simulation study. Clinical Neurophysiology, in press, 2002

KonOPACKI J, BLAND BH and ROTH SH. Evidence that activation of in vitro hippocampal theta rhythm only involves muscarinic receptors. Brain Research, 455: 110-114, 1988.

Krause CM, SillanmÄKi L, HÄGGQvist A and Heino R. Test-retest consistency of the event-related desynchronization/event-related synchronization of the 4-6, 6-8, 8-10 and 10-12 Hz frequency bands during a memory task. Clinical Neurophysiology, 112: 750-757, 2001.

Krause CM, Sillanmäki L, Koivisto M, SaArela C, Häggqvist A, Laine M and Hämäläinen H. The effects of memory load on event-related EEG desynchronization and synchronization. Clinical Neurophysiology, 111: 2071-2078, 2000.

KUTAS M and HILLYARD SA. Reading senseless sentences: Brain potentials reflect semantic incongruity. Science, 207: 203-205., 1980

Lachaux JP, Rodriguez E, Le Van Quyen M, Lutz A, Martinerie J and Varela FJ. Studying single trials of phase synchronous activity in the brain. International Journal of Bifurcation and Chaos, 10: 2429-2439, 2000.

Lachaux JP, Rodriguez E, Martinerie J and Varela FJ. Measuring phase synchrony in brain signals. Human Brain Mapping, 8: 194-208, 1999 
LEUNG L-WS and BorsT JGG. Electrical activity of the cingulate cortex. I. Generating mechanisms and relations to behavior. Brain Research, 407: 68-80, 1987.

LOPES DA SILVA FH. Neural mechanisms underlying brain waves: From neural membranes to networks. Electroencephalography and Clinical Neurophysiology, 79: 81-93, 1991a.

LoPES DA SILVA FH. Hippocampal RSA in humans. Trends in Neuroscience, 14: 138, $1991 \mathrm{~b}$.

LOPES DA SILVA FH. The rhythmic slow activity (theta) of the limbic cortex: Mechanisms of generation, models and functional implications. In TH Bullock and E Basar (Eds), Induced Rhythms in the Brain. Cambridge, MA: MIT Press, 1992, pp. 83-102.

Lopes da Silva FH, Witter MP, Boeijinga PH and Lohman AHM. Anatomic organization and physiology of the limbic cortex. Physiological Reviews, 70: 453-511, 1990.

Maguire EA, Burke T, Phillips J and Staunton H. Topographical disorientation following unilateral temporal lobe lesions in humans. Neuropsychologia, 34: 993-1001, 1996.

Malkova L and Mishrin M. Memory for locations of objects after separate lesions of the hippocampus and parahippocampal cortex in rhesus monkey. Society for Neuroscience Abstracts, 23: 12, 1997.

McEvoy LK, Pelloucoud E, Smith ME and Gevins A. Neurophysiological signals of working memory in aging. Cognitive Brain Research, 11: 363-376, 2001.

Mesulam MM. From sensation to cognition. Brain, 121: 1013-1052, 1998.

Meunier M, Bachevalier J, Mishin M and Murray EA. Effects on visual recognition of combined and separate ablations of the entorhinal and perirhinal cortex in rhesus monkeys. Journal of Neuroscience, 13: 5418-5432, 1993.

Miller R. Cortico-hippocampal Interplay and the Representation of Contexts in the Brain. Berlin: Springer-Verlag, 1991.

MulHolland T. The concept of attention and the electroencephalographic alpha rhythm. In CR Evans and TB Mulholland (Eds), Attention in Neurophysiology. Butterworth: London, 1969, pp. 100-127.

Munte TF, MatzKe M and Johannes S. Brain activity associated with syntactic incongruencies in words and pseudowords. Journal of Cognitive Neuroscience, 9: 300-311, 1997.

Nunez PL, Srinivasan R, Wetdorp AF, Wijesinghe RS, Tucker DM, Silberstein RB and Cadusch PJ. EEG coherency I: statistics, reference electrode, volume conduction, Laplacians, cortical imaging and interpretation at multiple scales. Electroencephalography and Clinical Neurophysiology, 103: 499-515, 1997.

O'KeEFE J and RECCE M. Phase relationship between hippocampal place units and the EEG theta rhythm. Hippocampus, 3: 317-330, 1993.

Petsche H, Stumpf C and Gogolak G. The significance of the rabbit's septum as a relay station between the midbrain and the hippocampus. The control of hippocampus arousal activity by septum cells. Electroencephalography and Clinical Neurophysiology, 14: 202-211, 1962.

PFURTSCHELlER G. Event-related synchronization (ERS): An electrophysiological correlate of cortical areas at rest. Electroencephalography and Clinical Neurophysiology, 83: 62-69, 1992.

PFURTSCHELlER G and ARANIBAR A. Event-related cortical desynchronization detected by power measurements of scalp EEG. Electroencephalography and Clinical Neurophysiology, 42: 817-826, 1977.

Pfurtscheller G and Lopes DA Silva FH. Event-related EEG-MEG synchronization and desynchronization: Basic principles. Clinical Neurophysiology, 110: 1842-1857, 1999.

Pulvermuller F. Words in the brain's language. Behavioural and Brain Sciences, 22: 253-336, 1999.

Rizzuto D, Aschenbrenner-Scheibe R, Schulze-Bonhage A, Freiman T, Madsen JR and Kahana MJ. Memory retrieval operations produce a reset of theta oscillations in humans. Journal of Cognitive Neuroscience, in press (abstract), 2001.

Rodriguez E, George N, Lachaux JP, Martinerie J, Renault B and Varela FJ. Perception's shadow: Long-distance synchronization of human brain activity. Nature, 397: 430-433, 1999.

Roelfsema PR, Engel AK, Konig P and Singer W. Visuomotor integration is associated with zero time-lag synchronization among cortical areas. Nature, 385: 157-161, 1997.

Routtenberg A and TAub F. Hippocampus and superior colliculus: Congruent EEG activity demonstrated by a simple measure. Behavioural Biology, 8: 801-805, 1973.

SLAWINSKa U and KASICKI S. Theta-like rhythm in depth EEG activity of hypothalamic areas during spontaneous or electrically induced locomotion in rats. Brain Research, 678: 117-126, 1995.

STERIADE M. Synchronized activities of coupled oscillators in the cerebral cortex and thalamus at different levels of vigilance. Cerebral Cortex, 7: 583-604, 1997.

Steriade M, Gloor P, Llinas RR, Lopes da Silva FH and Mesulam MM. Basic mechanisms of cerebral rhythmic activities. Electroencephalography and Clinical Neurophysiology, 76: 481-508, 1990.

STEWARD M and Fox SE. Do septal neurons pace the hippocampal theta rhythm? Trends in Neuroscience, 13: 163-168, 1990.

TALLON-BAUDRY C and BERTRAND O. Oscillatory gamma activity in humans and its role in object representation. Trends in Cognitive Science, 3: 151-162, 1999. 
Tallon-Baudry C, Bertrand O, Peronnet F and Pernier J. Induced gamma band activity during the delay of a visual short-term memory task in humans. Journal of Neuroscience, 18: 4244-4254, 1998.

TAYLOR SJ, McANDREws MP, MAKAREC K and MiKULIS D. Event-related fMRI reveals a synchronized hippocampal system associated with verbal encoding. NeuroImage, 13: S747, 2001.

TESCHE CD and KARHU J. Theta oscillations index human hippocampal activation during a working memory task. Proceedings of the National Academy of Sciences USA, 97: 919-924, 2000.

Treves A and Rolls ET. Computational analysis of the role of the hippocampus in memory. Hippocampus, 4: 374-391, 1994.

Vinogradova OS, BRAHZHNIK ES, Karanov AN and Zhadina SD. Analysis of neuronal activity in rabbit's septum with various conditions of deafferentation. Brain Research, 187: 354-368, 1980.

Van Turennout M, Hagoort P and Brown CM. Brain activity during speaking: From syntax to phonology in 40 milliseconds. Science, 280: 572-574, 1998.

Von STEIN A and SARNTHEIN J. Different frequencies for different scales of cortical integration: From local gamma to long-range alpha/theta synchronization. International Journal of Psychophysiology, 38: 301-313, 2000.

WANG XJ and BuZSAKI G. Gamma oscillation by synaptic inhibition in a hippocampal interneuronal network model. Journal of Neuroscience, 16: 6402-13, 1996.

WeISS S, MULLER HM and RAPPELSBERGER P. theta synchronization predicts efficient memory encoding of concrete and abstract nouns. Neuroreport, 11:2357-2361, 2000.

Whittington MA, Traub RD, Kopell N, ERmentrout B and BuHl EH. Inhibition-based rhythms: experimental and mathematical observations on network dynamics. International Journal of Psychophysiology, 38: 315-36, 2000

WINSON J. Loss of hippocampal theta rhythm results in spatial memory deficit in the rat. Science, 201: 160-163, 1978.

Witter MP, Naber PA, van Haeften T, Machielsen WCM, Rombouts SARB, Barkhof F, SCHELTENS P and LoPeS DA SILvA FH. Cortico-hippocampal communication by way of parallel parahippocampal-subicular pathways. Hippocampus, 10: 398-410, 2000.

Marcel C.M. Bastiaansen, FC Donders Centre for Cognitive Neuroimaging, Trigon 181, PO Box 9101, 6500 HB Nijmegen, The Netherlands.

e-mail: marcel.bastiaansen@fcdonders.kun.nl 